\title{
Functional Status and Liver Disease Phenotype: Frailty, Thy Presence Is Ominous
}

\author{
Manhal Izzy ${ }^{1}$ Alexandra Shingina ${ }^{1}$
}

Published online: 10 December 2019

○) Springer Science+Business Media, LLC, part of Springer Nature 2019

With the continued shortage of organs available for liver transplantation (LT), the primary priority of the LT community is optimization of the utilization of this scarce resource, a priority that has triggered multiple studies over the past several years in order to identify extrahepatic pathologies and comorbidities that may relate positively or negatively to LT outcomes. Consequently, frailty and sarcopenia, closely associated yet nonequivalent conditions, have emerged as disorders that can significantly impair pre- and post-LT outcomes [1]. They are, however, not considered conventional surrogates of advanced liver disease such as the Model for End-Stage Liver Disease (MELD) score. The diagnosis of sarcopenia, defined as the loss of muscle mass, requires objective muscle mass assessment. On the other hand, frailty is a systemic impairment associated with increased vulnerability to stressors and decrease in functional reserve. In view of this definition, multiple subjective and objective tools have been used to assess frailty [1]. Nevertheless, the only tool for frailty or functional reserve assessment that is currently captured in United Network for Organ Sharing (UNOS) database is the Karnofsky Performance Status Scale (KPSS), which has been available from 2005 onward.

The KPSS was originally developed to quantify "subjective improvement" in lung cancer patients treated with experimental nitrogen mustard in 1948; these patients would suffer from toxic effects of nitrogen mustard but showed overall "objective improvement" in cancer such as decreased lesion size at the expense of patient reported adverse symptoms [2]. Over the past 70 years, it has been widely used in multiple disciplines, including clinical trials, in order to assess the overall performance status of the patient [3, 4]. KPSS is a subjective assessment approach that is usually administered by the provider utilizing patient's own

Alexandra Shingina

alexandra.shingina@vumc.org

1 Division of Gastroenterology, Hepatology and Nutrition, Vanderbilt University Medical Center, Nashville, TN, USA reporting of extent of performance of daily activities. It is graded on a $0-100 \%$ scale (patients with normal activity $100 \%$, death $0 \%$ ) and is divided into 11 categories (by increments of 10) and occasionally into 4 categories (by increments of 20) in order to simplify measurements and correlate with other published performance status scales. As an example, a patient with a KPSS of $80 \%$ can carry out normal activity with effort and exhibits some signs and symptoms of the disease, whereas a patient with KPSS of $40 \%$ is deemed disabled and requires special care and assistance. With 4 categories, however, category 1 (KPSS1) refers to healthiest patients with KPSS $80-100 \%$ and category 4 (KPSS4) to patients with worst functional status at 10-30\%.

In the current issue of Digestive Diseases and Sciences, McCabe et al. [5] explore the effect of reported KPSS on waitlist and post-LT mortality in 94,201 patients listed for LT from 2005 to 2017, stratified by selected etiologies of liver disease [nonalcoholic steatohepatitis (NASH) vs. chronic hepatitis $\mathrm{C}(\mathrm{HCV})$ vs. alcohol-related liver disease (ALD) vs. combined HCV/ALD] using the UNOS database. ALD patients were more likely to have lower KPSS scores at the time of listing and LT when compared with other groups and having shorter waitlist to LT times compared with NASH patients. NASH and ALD patients had the highest waitlist mortality. ALD patients were also more likely to have higher median MELD score at listing and transplant (20 and 25, respectively) as compared with NASH patients (16 and 22, respectively), HCV patients (15 and 19, respectively), or HCV/ALD (17 and 22, respectively). ALD patients were also more likely to suffer from hepatic encephalopathy (HE) and moderate ascites. Lower KPSS scores were independently predictive of waitlist mortality across all disease etiologies, with a more pronounced effect on ALD and HCV patients. Conversely, the negative impact of lower KPSS scores on 5-year post-LT mortality was more pronounced in ALD and NASH. It is noteworthy that 5-year post-LT survival in patients with KPSS category 4 was inferior in patients with $\mathrm{HCV}$ or concurrent $\mathrm{HCV}$ and $\mathrm{ALD}$, 
likely owing to the recurrence of HCV and related complications prior to the advent of direct acting antiviral therapy.

The methodology of the study raises several concerns about potential biases. First, KPSS is a subjective assessment with inter- and intra-observer variability and, therefore, it has previously been described as suboptimal in non-cirrhotic populations [6]. In LT candidates, a study by Dunn et al. [7] demonstrated that KPSS is not reliable in reflecting the actual physical activity which was measured in the study via an accelerometer/thermal sensing armband. Second, previous study showed that lower KPSS scores reported were strongly associated with UNOS region, center size, market competition, likely due to bias owing to factors other than functional status [8]. Third, assessment of functional status in cirrhotic populations is profoundly influenced by the incidence of HE, which was not adjusted for in the current analysis. Finally, as ALD patients seemed to have higher MELD scores at listing and LT, the results should be adjusted for ICU admission status, which would also greatly influence KPSS assessment. In terms of post-LT outcomes, several variables that could influence outcomes were not reported or incorporated in the analysis, such as donor data, dialysis requirements, LT center volume, other etiologies of liver disease, and relatively common post-LT comorbidities related to mortality such as cardiovascular disease and postLT malignancy.

Contrary to the current study, where lower KPSS was seen in patients with ALD, two other recent studies utilizing other assessment tools have reported that the underlying liver disease most associated with frailty is in fact NASH $[9,10]$. One of these studies objectively assessed frailty and functional status using hand grip, timed chair stands, and balance testing. It also showed that HCV patients are least affected by frailty [9]. The second study employed the Rockwood frailty index, which evaluates subjective and objective variables, showed that while frailty was more common in $\mathrm{NASH}$, sarcopenia (using radiologic muscle assessment) was more common in ALD. With multivariate analysis, neither frailty nor sarcopenia among ALD patients impacted delisting [10]. These observations validate the aforementioned concerns about the limited reliability of KPSS. The more pronounced impact of KPSS on post-LT survival in patients with NASH and ALD in the McCabe et al. study could have been confounded by the systemic comorbidities commonly observed in NASH and ALD patients.

Another valuable aspect of this study is that it illustrates the burden of illness in listed patients with alcohol-related cirrhosis as evident by higher listing and LT MELD and a higher possibility of decompensation manifestations, potentially reflecting late clinical presentation of these patients as well as delayed listing until requirements of sobriety are achieved. Publication of the seminal prospective multicenter study by Mathurin et al. [11] on LT for alcoholic hepatitis has opened another frontier for early LT in ALD. Early results in 147 patients in the USA who underwent early LT for severe AH showed similar survival rates to patients transplanted for other indications [12]. In fact, recently published AASLD guidance on ALD management suggests consideration of early LT in "carefully selected patients with favorable psychosocial profiles" in severe AH not responding to medical therapy [13]. Thus, the number of ALD patients that the LT community will be evaluating for early LT will likely be growing in the coming years.

This study represents a valuable attempt toward defining the differential impact of frailty and poor functional status among several etiologies of liver disease on pre- and postLT clinical outcomes. The findings certainly call for further studies addressing this issue and using objective assessments of functional status and with a prospective design. Identifying liver disease characteristics that can be associated with impaired pre- and post-LT outcomes is essential to the identification and management of high-risk patients. In case of frailty, a holistic approach involving early reliable identification, extensive dietary interventions, physical therapy, and treatment of decompensation manifestations may reverse the impact of frailty on outcomes and potentially improve postLT survival in order to ensure better utilization of scarce organs.

\section{References}

1. Bhanji RA, Carey EJ, Yang L, Watt KD. The long winding road to transplant: how sarcopenia and debility impact morbidity and mortality on the waitlist. Clin Gastroenterol Hepatol. 2017;15:1492-1497.

2. Timmermann C. 'Just give me the best quality of life questionnaire': the Karnofsky scale and the history of quality of life measurements in cancer trials. Chronic Illn. 2013;9:179-190.

3. Yates JW, Chalmer B, McKegney FP. Evaluation of patients with advanced cancer using the Karnofsky performance status. Cancer. 1980;45:2220-2224.

4. Mor V, Laliberte L, Morris JN, Wiemann M. The Karnofsky Performance Status Scale. An examination of its reliability and validity in a research setting. Cancer. 1984;53:2002-2007.

5. McCabe P, Galoosian A, Wong RJ. Patients with alcoholic liver disease have worse functional status at time of liver transplant registration and greater waitlist and post-transplant mortality which is compounded by older age. Dig Dis Sci. (Epub ahead of print). https://doi.org/10.1007/s10620-019-05891-1.

6. Chow R, Chiu N, Bruera E, et al. Inter-rater reliability in performance status assessment among health care professionals: a systematic review. Ann Palliat Med. 2016;5:83-92.

7. Dunn MA, Josbeno DA, Schmotzer AR, et al. The gap between clinically assessed physical performance and objective physical activity in liver transplant candidates. Liver Transplant. 2016;22:1324-1332.

8. Wang CW, Lai JC. Reporting functional status in UNOS: The weakness of the Karnofsky Performance Status Scale. Clin Transplant. 2017;31:e13004. 
9. Lai JC, Rahimi RS, Verna EC, et al. Frailty associated with waitlist mortality independent of ascites and hepatic encephalopathy in a multicenter study. Gastroenterology. 2019;156:1675-1682.

10. Bhanji RA, Narayanan P, Moynagh MR, et al. Differing impact of sarcopenia and frailty in nonalcoholic steatohepatitis and alcoholic liver disease. Liver Transplant. 2019;25:14-24.

11. Mathurin P, Moreno C, Samuel D, et al. Early liver transplantation for severe alcoholic hepatitis. N Engl J Med. 2011;365:1790-1800.

12. Lee BP, Terrault NA. Early liver transplantation for severe alcoholic hepatitis: moving from controversy to consensus. Curr Opin Organ Transplant. 2018;23:229-236.
13. Lucey MR, Im GY, Mellinger JL, Szabo G, Crabb DW. Introducing the 2019 American Association for the study of liver diseases guidance on alcohol-related liver disease. Liver Transpl. 2019. https://doi.org/10.1002/lt.25600.

Publisher's Note Springer Nature remains neutral with regard to jurisdictional claims in published maps and institutional affiliations. 